\title{
Estudo bibliométrico da produção científica em endodontia
}

\author{
Bibliometric study of scientific production in Endodontics \\ Estudio bibliometrico de la producción científica en Endodoncia \\ Kaiane Tavares PONTES ${ }^{1}$ \\ Everton Lindolfo SILVA ${ }^{1}$ \\ Robeci Alves MACÊDO FILHO ${ }^{1}$ \\ Diego Romário SILVA ${ }^{2}$ \\ Francisco Jadson LIMA ${ }^{3}$ \\ ${ }^{1}$ Graduado(a) em Odontologia, Universidade Estadual da Paraíba - UEPB, 58233-000, Araruna-PB, Brasil. \\ ${ }^{2}$ Doutorando em Farmacologia, Anestesiologia e Terapêutica, Faculdade de Odontologia de Piracicaba - FOP/UNICAMP, \\ Universidade Estadual de Campinas 13414-018 Piracicaba-SP, Brasil. \\ ${ }^{3}$ Mestrado em Odontologia pela Universidade Estadual da Paraíba - UEPB 58233-000 Araruna-PB, Brasil, \\ Doutorando em Patologia Oral, Universidade Federal do Rio Grande do Norte - UFRN 59080-000 Natal-RN, Brasil
}

\begin{abstract}
Resumo
Objetivo: Realizar um estudo bibliométrico dos resumos da SBPqO, traçando um perfil dos trabalhos publicados. Material e métodos: Realizou-se um estudo descritivo, analisando os resumos publicados em anais do referido evento. Os trabalhos foram classificados de acordo com ano de publicação; região; estado; instituição; tipo de instituição; objeto, área ou assunto estudado; tipo de estudo; caracterização do estudo; citação de submissão ao Comitê de Ética e Pesquisa; financiamento para realização da pesquisa; agência/órgão de fomento. A coleta foi realizada por três examinadores, que organizaram os resultados em um banco informatizado. Resultados: De um total de 8.381 resumos, 743 (8,86\%) foram referentes à área endodôntica, sendo a maioria desenvolvida na região sudeste (66,1\%), principalmente no estado de São Paulo (47\%). Como assunto mais pesquisado destacou-se "limas, materiais e instrumentos", com 47,6\%. Observou-se maior quantidade de estudos experimentais $(66,1 \%)$, sendo os do tipo in vitro mais presentes (64,3\%). A maioria das pesquisas foi advinda de instituições públicas $(73,8 \%)$, principalmente de duas instituições específicas: USP com 14,8\% e da UNESP com 13,5\% dos trabalhos. Percebeu-se, ainda, uma grande quantidade de estudos realizados sem auxílio financeiro (68,5\%). Não houve citação de submissão ao Comitê de Ética e Pesquisa. Conclusão: Constatou-se que existe o interesse científico pela área endodôntica mesmo que em pequena escala. Ao mesmo tempo, observou-se a necessidade de maior incentivo às pesquisas em alguns locais, visto que houve concentração em determinadas regiões e instituições, bem como uma diminuição das pesquisas ao considerar-se os anos analisados.
\end{abstract}

Descritores: Pesquisa em Odontologia; Bibliometria; Endodontia.

\begin{abstract}
Objective: This study aimed to perform a comparative analysis of self-perception, the behaviors and attitudes of oral health and the oral health status of adult patients treated at the clinics of the Dentistry Graduate Course from the Dentristy Department of the Federal University of Rio Grande do Norte - UFRN. Methods: A sample of 36 patients was evaluated by applying a questionnaire about self-perception and oral hygiene habits, besides the evaluation of oral health status based on clinical examination data and obtaining the oral indices such as DMF, IVP and IGB. The Data was analyzed using descriptive statistics. Results: It was observed that $94.4 \%$ of patients reported having a poor oral health and only $5.6 \%$ believed they had adequate oral health. It was found that $41.7 \%$ of patients showed low DMFT and $21(58.3 \%)$ high DMFT. A significant proportion of patients (61.8\%) felt that having a poor oral health showed high DMFT. Most of the sample felt that having a poor gum health and VPI had a high moderate to severe degree of gingival inflammation. Conclusion: There was a correlation between selfrated oral health and oral clinical conditions of the population evaluated. The results suggest that self-perception of oral health may represent a useful tool in dentistry to determine characteristics of the major dental problems of a population and the need for the same therapeutic approach, which may represent a potentially powerful and useful to test the transfer of information from professionals for the population and thus the effectiveness of dental treatment.

Descriptors: Dental Research; Bibliometrics; Endodontics.
\end{abstract}

\section{Resumen}

Objetivo: Realizar un estudio bibliométrico de los resúmenes de la SBPqO, trazar un perfil de los trabajos publicados. Material e métodos: Realizou-se un estudio descritivo, analizar los resúmenes publicados en anais do referido evento. Los trabajos se han clasificado de acuerdo con ano de publicación; Region Estado; Institución Tipo de institution; Objeto, área o documento estudiado; Tipo de estudio; Caracterização del estudio; Citación de sumisión al Comitê de Ética y Búsqueda; Financiamiento para la investigación; Agência / órgão de fomento. A coleta fue realizada por tres examinadores, que organizó los resultados en un banco informatizado. Resultados: De um total de 8.381 resúmenes, $743(8,86 \%)$ fueron referenciados a área endodóntica, siendo una mayoría desarrollada en la región sudeste (66,1\%), principalmente no estado de São Paulo (47\%). Como consultado más pesquisado destacou-se "limas, materiales e instrumentos", com 47,6\%. Observou-se mayor cantidad de estudios experimentales $(66,1 \%)$, siendo el tipo in vitro más presentes $(64,3 \%)$. (73,8\%), principalmente de dos empresas específicas: USP com 14,8\% e da UNESP com 13,5\% de los trabajos. Percebeu-se, todavía, una gran cantidad de estudios realizados sin auxílio financiero $(68,5 \%)$. No existe una cita de la sumisión al Comite de Ética y Búsqueda. Conclusión: Constatou-se que existe el interés científico en el área endodóntica que en menor escala. Al mismo tiempo, observamos una necesidad de mayor incentivo para las investigaciones en algunos lugares, hemos visto que hay una concentración en las regiones y las instituciones.

Descriptores: Investigación Dental; Bibliometría; Endodoncía.

\section{INTRODUÇÃO}

A Endodontia é a área da Odontologia que abrange o estudo da morfologia, fisiologia e patologia da polpa dentária humana e dos tecidos periapicais. $\mathrm{O}$ seu estudo engloba ciências básicas e clínicas, incluindo a biologia da polpa normal, etiologia, diagnóstico, prevenção, tratamento das doenças, lesões pulpares e/ou periapicais ${ }^{1}$. Em todas as 
áreas do conhecimento, as revisões da literatura e os estudos bibliométricos funcionam como fonte de evidência para caracterizar a evolução dos estudos que estão sendo realizados.

A bibliometria, como área de estudo da ciência da informação, tem um papel fundamental na análise da produção científica, pois seus indicadores retratam o grau de desenvolvimento de uma área do conhecimento de um campo científico ou de saber ${ }^{2}$. Sendo assim, uma das formas de se fazer avaliação da produção científica é através da utilização de métodos que permitam medir a produtividade dos pesquisadores, grupos ou instituições de pesquisa ${ }^{3}$. As pesquisas possuem considerável importância entre as atividades realizadas nas universidades e instituições de ensino, pois permitem a ampliação dos conhecimentos. É responsabilidade da produção acadêmica disseminar o saber, tornando-o público ${ }^{4}$.

As pesquisas brasileiras, principalmente relacionadas à Odontologia, são muito respeitadas na comunidade científica internacional e demostram um aumento expressivo na sua produção nos últimos anos ${ }^{5,6}$. Este crescimento não é homogêneo, apresentando diferenças no mesmo país quanto às suas regiões, bem como entre áreas de pesquisa ${ }^{7}$.

A Sociedade Brasileira de Pesquisa Odontológica $(\mathrm{SBPqO})$ realiza anualmente uma reunião para discutir pesquisas em Odontologia. São apresentados estudos previamente selecionados, oriundos de todas as regiões do país e que abrangem as diversas áreas da Odontologia, sendo possível tomá-los como representativos do conjunto da produção científica no Brasil ${ }^{8}$.

O conhecimento científico deve ser apresentado para que a Comunidade Científica possa avaliá-lo e, assim, poder cumprir sua finalidade social. Em virtude disso, este trabalho teve por objetivo realizar um levantamento bibliométrico dos estudos realizados na área da Endodontia no Brasil, utilizando os dados publicados nos anais da $\mathrm{SBPqO}$, que é considerado o maior evento da comunidade científica odontológica do país.

\section{MATERIAL E MÉTODO}

\section{- Caracterização do estudo}

Esta pesquisa é classificada como descritiva, uma vez que buscou descrever o perfil da produção científica na área da Endodontia. Trata-se de uma pesquisa documental e observacional, uma vez que foi realizada com base em dados secundários. A população deste estudo foi constituída por todos os resumos/trabalhos publicados nos Anais das Reuniões da SBPqO dos anos de 2011, 2013 e 2014.

\section{○ Amostra}

A amostra deste trabalho foi obtida após leitura de todos os resumos/trabalhos publicados nos Anais das Reuniões da SBPqO referentes aos anos de 2011, 2013 e 2014. Os trabalhos publicados no ano de 2012 foram excluídos da pesquisa devido à sobreposição da reunião da $\mathrm{SBPqO}$ neste ano a outro evento cientifico internacional, a International Association for Dental Research (IADR), tendo por consequência junção das pesquisas internacionais e nacionais, terminando por fugir ao objetivo principal do presente estudo, que visa, em princípio, caracterizar a pesquisa na área de endodontia no Brasil.

Para seleção dos resumos, três pesquisadores previamente calibrados, revisaram trabalho a trabalho, lendo-os e selecionando aqueles que tinham como objeto ou área de estudo principal ou relacionada à Endodontia, considerando para seleção final o respeito aos critérios de inclusão e exclusão para a pesquisa (Quadro 1). Após a aplicação de tais critérios, a seleção final compôs uma amostra de 743 resumos.

Quadro 1 - Distribuição e definição dos critérios utilizados para seleção amostral. Araruna, PB (2016)

\begin{tabular}{|l|ll|}
\hline \multicolumn{3}{|c|}{ Critérios utilizados para Seleção Amostral } \\
\hline \multicolumn{2}{|c|}{ Critérios de Inclusão } & \multicolumn{3}{|c|}{ Critérios de Exclusão } \\
\hline $\begin{array}{l}\text { Tema principal ou } \\
\text { relacionado à Endodontia }\end{array}$ & $\begin{array}{l}\text { Não ter relação com a } \\
\text { Endodontia }\end{array}$ \\
\hline Trabalho de Pesquisa & $\begin{array}{l}\text { Trabalhos de Extensão, } \\
\text { Relatos de Experiência }\end{array}$ \\
\hline $\begin{array}{l}\text { Publicação do resumo } \\
\text { disponível }\end{array}$ & $\begin{array}{l}\text { Publicação do resumo } \\
\text { indisponível }\end{array}$ \\
\hline $\begin{array}{l}\text { Ser apresentação Oral ou } \\
\text { Painel }\end{array}$ & $\begin{array}{l}\text { Ser apresentação sob outras } \\
\text { formas }\end{array}$ \\
\hline
\end{tabular}

Após seleção dos resumos pelos três pesquisadores, foi realizado o teste de Kappa para verificação da concordância interexaminadores, obtendo-se um valor de 0.83 (acordo quase perfeito).

\section{- Coleta de dados}

Após a seleção dos resumos, os pesquisadores leram novamente cada um dos trabalhos, e realizaram a análise bibliométrica, que segundo Guedes et al. ${ }^{9}$, quantifica, descreve e prediz o processo de comunicação escrita.

Para essa análise foi elaborada uma ficha específica para cada resumo contendo as seguintes variáveis: ano de publicação; região de origem da pesquisa; estado de origem da pesquisa; instituição de origem da pesquisa; tipo de instituição de origem da pesquisa (pública ou privada); objeto, área ou assunto estudado; tipo de estudo realizado; caracterização do estudo realizado; citação de submissão ao Comitê de Ética e Pesquisa (CEP); financiamento para realização da pesquisa; agência/órgão de fomento.

E por fim, os 743 fichamentos foram dispostos em uma tabela informatizada criada em ambiente Excel Microsoft para facilitar a análise quantitativa das variáveis.

\section{- Análise dos dados}

Após a coleta, os resultados obtidos foram organizados em um banco de dados informatizado com o auxílio do pacote estatístico Statistical Package for the Social Sciences (SPSS) (v.20). A análise da distribuição dos dados foi realizada de forma descritiva expondo os resultados em porcentagens, frequências simples e relativa.

\section{RESULTADOS}

De um total de 8.381 estudos publicados nos anais das reuniões da SBPqO nos anos de 2011, 2013 e 2014 foram encontrados $743(8,86 \%)$ trabalhos relacionados com a área da Endodontia. Observou-se uma diminuição na quantidade de trabalhos dessa natureza, uma vez que em 2011 verificou-se $283(38,1 \%)$ trabalhos publicados, em 2013 o número diminuiu para 255 (34,3\%), chegando a 205 $(27,6 \%)$ em 2014, conforme demonstrado na Figura 1.

Em relação à distribuição por região desses trabalhos, houve maior concentração das pesquisas provindas da região sudeste com $491(66,1 \%)$ resumos, destacando-se o estado de São Paulo que foi responsável por $349(47 \%)$ dos estudos publicados. Em seguida, verificam-se as regiões sul e centro-oeste, com $137(18,4 \%)$ e $53(7,1 \%)$ estudos, respectivamente, como apresentado nas Tabela 1 e 2 . 


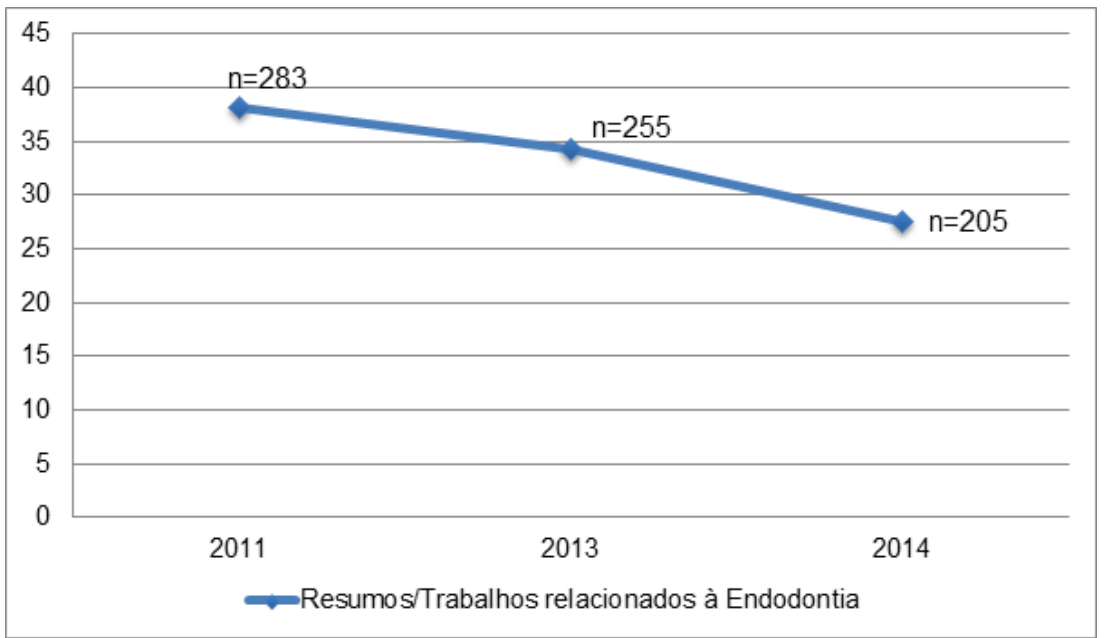

Figura 1: Distribuição absoluta e percentual dos trabalhos publicados nos anais da SBPqO dos anos de 2011, 2013 e 2014.

Tabela 1. Distribuição das pesquisas segundo as regiões do território nacional nos anais da SBPqO dos anos de 2011, 2013 e 2014

\begin{tabular}{|c|c|c|}
\hline \multirow{2}{*}{ REGIÕES } & \multicolumn{2}{|c|}{ FREQUÊNCIA } \\
\hline & $\mathbf{n}$ & $\%$ \\
\hline Sudeste & 491 & 66,1 \\
\hline Sul & 137 & 18,4 \\
\hline Centro-Oeste & 53 & 7,1 \\
\hline Nordeste & 45 & 6,1 \\
\hline Norte & 17 & 2,3 \\
\hline Total & 743 & 100,0 \\
\hline
\end{tabular}

Tabela 2. Distribuição das pesquisas segundo os estados do território nacional nos anais da SBPqO dos anos de 2011, 2013 e 2014

\begin{tabular}{l|c|c}
\multirow{2}{*}{ ESTADOS } & \multicolumn{2}{c}{ FREQUENCIA } \\
\cline { 2 - 3 } & $\mathrm{n}$ & $\%$ \\
\hline Amazonas & 4 & 0,5 \\
\hline Bahia & 6 & 0,8 \\
\hline Ceará & 12 & 1,6 \\
\hline Distrito-Federal & 3 & 0,4 \\
\hline Espirito Santo & 1 & 0,1 \\
\hline Goías & 3 & 1,1 \\
\hline Maranhão & 67 & 0,4 \\
\hline Minhas Gerais & 10 & 9,0 \\
\hline Mato Grosso do Sul & 33 & 1,3 \\
\hline Mato Grosso & 13 & 4,4 \\
\hline Pará & 9 & 1,7 \\
\hline Paraíba & 3 & 1,2 \\
\hline Piauí & 32 & 0,4 \\
\hline Paraná & 74 & 4,3 \\
\hline Rio de Janeiro & 3 & 10,0 \\
\hline Rio Grande do Norte & 75 & 0,4 \\
\hline Rio Grande do Sul & 29 & 10,1 \\
\hline Santa Catarina & 3 & 3,9 \\
\hline Sergipe & 349 & 0,4 \\
\hline São Paulo & 743 & 47,0 \\
\hline & & 100,0 \\
\hline
\end{tabular}

Entre os assuntos ou temas mais pesquisados destacou-se a categoria referente a "limas, materiais e instrumentais", com 354 (47,6\%) estudos, seguida da categoria "medicação, substância e irrigação" que consistiu em $181(24,4 \%)$ trabalhos. Outros temas que investigaram seguimentos como patologias pulpoperiapicais, tratamento e técnicas de preparo, obturação, retratamento, avaliações de tratamentos e microbiologia podem ser vistos na Figura 2.

Quanto ao tipo de estudo mais desenvolvido, os de cunho experimental representaram 712 (95,8\%) dos trabalhos, seguido dos observacionais e revisões com 29 $(3,9 \%)$ e 2 (0,3\%), respectivamente. Dos estudos experimentais, as pesquisas in vitro foram as mais frequentes, consistindo em $478 \quad(64,3 \%)$ trabalhos (Tabela 3).

Quanto às instituições de origem dos trabalhos é importante destacar que $548(73,8 \%)$ destes foram advindos de instituições públicas, havendo predomínio de duas instituições, a Universidade Estadual de São Paulo (USP) com $110(14,8 \%)$ trabalhos, seguida da Universidade Estadual Paulista (UNESP) com 100 (13,5\%) trabalhos. Outras 64 instituições, entre públicas e privadas, dividiram a autoria dos trabalhos restantes (Tabela 4).

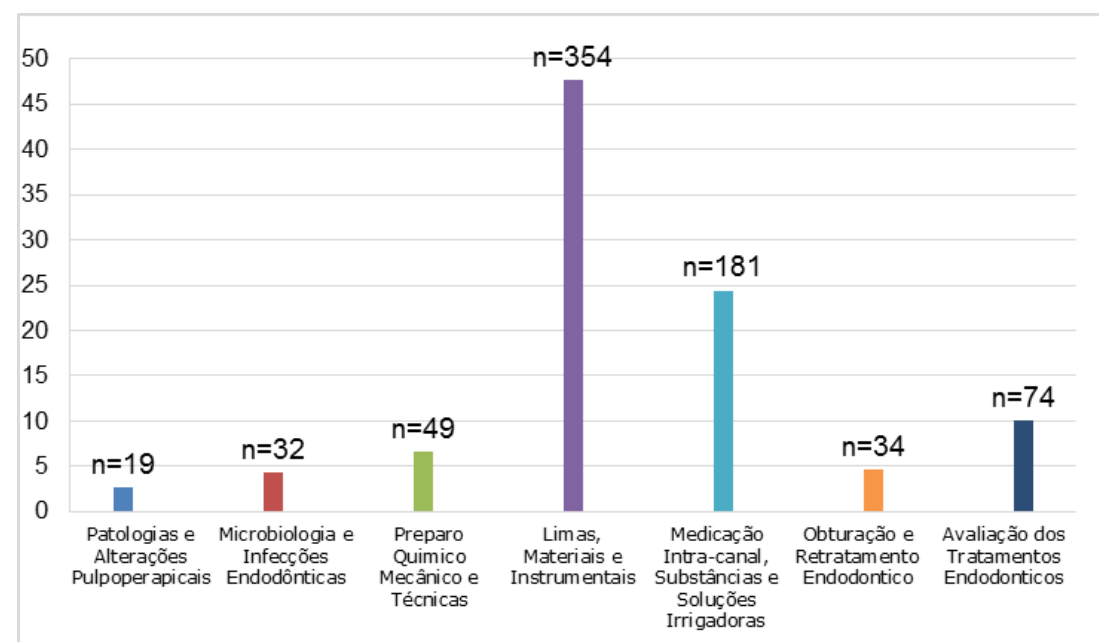

Figura 2: Distribuição das pesquisas segundo os assuntos/temas estudados nos anais da SBPqO dos anos de 2011, 2013 e 2014.

Tabela 3. Distribuição das pesquisas segundo o tipo e caracterização do estudo nos anais da SBPqO dos anos de 2011, 2013 e 2014.

\begin{tabular}{|c|c|c|}
\hline \multirow[b]{2}{*}{ Tipo de Estudo } & \multicolumn{2}{|c|}{ FREQUÊNCIA } \\
\hline & $\mathrm{n}$ & $\%$ \\
\hline Experimental & $712^{*}$ & 95,8 \\
\hline Observacional & 29 & 3,9 \\
\hline Documental/Revisão & 2 & 0,3 \\
\hline Total & 743 & 100,0 \\
\hline
\end{tabular}

Tabela 4. Distribuição das pesquisas segundo a instituição de origem e se pública ou privada nos anais da SBPqO dos anos de 2011, 2013 e 2014

\begin{tabular}{|c|c|c|}
\hline \multirow[t]{2}{*}{ Tipo de Instituição } & \multicolumn{2}{|c|}{ FREQUÊNCIA } \\
\hline & $\mathrm{n}$ & $\%$ \\
\hline Pública & 548 & 73,8 \\
\hline Privada & 195 & 26,2 \\
\hline Total & 743 & 100,0 \\
\hline \multirow[t]{2}{*}{ Instituição de Origem } & \multicolumn{2}{|c|}{ FREQUÊNCIA } \\
\hline & $\mathrm{n}$ & $\%$ \\
\hline USP & 110 & 14,8 \\
\hline UNESP & 100 & 13,5 \\
\hline Outras (64 instituições) & 533 & 71,7 \\
\hline Total & 743 & 100,0 \\
\hline
\end{tabular}

Em relação ao auxílio financeiro, constatou-se que mais da metade das pesquisas não receberam apoio de qualquer instituição fomentadora de pesquisa 509 (68,5\%), enquanto apenas $234(31,5 \%)$ relataram apoio financeiro. Dentre as instituições que fomentaram as pesquisas destacaram-se as Fundações de Amparo à Pesquisa (FAPs) com $175 \quad(51,2 \%)$, o Conselho Nacional de Desenvolvimento Científico e Tecnológico (CNPq) com 81 (33,4\%), e a Coordenação de Aperfeiçoamento de Pessoal de Nível Superior (CAPES) com $39(15,4 \%)$. Quanto às fundações, estas representam apoios estaduais e institucionais.

Deve-se destacar que não houve citação de submissão ao CEP em nenhum dos resumos/trabalhos.

\section{DISCUSSÃO}

Uma análise bibliométrica representa um importante instrumento no diagnóstico e monitoramento da divulgação do conhecimento científico em determinada área ${ }^{10}$. Neste trabalho, constatou-se uma grande contribuição de estudos 
endodônticos para a produtividade científica na Odontologia. Entretanto, o que se pode observar foi uma diminuição desses estudos com o passar dos anos, fato que deve ser cuidadosamente abordado pela classe em busca da continuidade na produção de conhecimento cientifico.

A concentração das pesquisas provindas da região sudeste, referente a $491(66,1 \%)$ resumos, principalmente no estado de São Paulo, concorda com os dados de BritoJúnior et al. ${ }^{10}$ que constataram que São Paulo foi o estado com maior produtividade, sendo responsável por $53,9 \%$ do total de trabalhos das publicações pesquisadas por eles, acontecidas entre 2008 e 2010. Assim, percebe-se que a pesquisa científica nas universidades de São Paulo é mais bem explorada que nas demais universidades do Brasil. Isso implica em maior quantidade de recursos e de resultados científicos para as universidades em questão.

$\mathrm{Na}$ categoria "assuntos/temas mais estudados" no estudo de Miller $^{11}$ houve uma parcela significativa da amostra $(27,41 \%)$ que não se enquadrava nos principais grupos de temas, sendo denominada "outros". Na sequência "casos clínicos" foi o tema de maior significância, com $19,14 \%$ dos estudos. Já Brito-Júnior et al. ${ }^{10}$ encontraram "materiais dentários" como tema mais prevalente dentre as publicações brasileiras no Journal of Endodontics e no International Endodontic Journal de 2008 a 2010, sendo referente a $38,6 \%$ da amostra. Em nosso trabalho, houve prevalência do tema "limas, materiais e instrumentos" $(24,4 \%)$. Observa-se, assim, que os temas mais pesquisados mudam de acordo com o estudo realizado, o que é interessante do ponto de vista científico.

Em relação à natureza das instituições, Brito-Júnior et al. ${ }^{10}$ constataram que $53,9 \%$ e $13,4 \%$ das publicações originaram-se, respectivamente, de instituições públicas e privadas e em 32,8\% delas houve parceria entre instituições das duas modalidades. Em nossa pesquisa, as instituições públicas consistiram na maioria dos estudos, $73,8 \%$. Dessa forma, é possível que as instituições públicas proporcionem maior suporte às pesquisas científicas, para seus alunos.

Sobre o auxílio financeiro, percebeu-se que a maior parte das pesquisas não recebeu apoio de instituições fomentadoras de pesquisa. Dentre aquelas que financiaram destacaram-se as FAPs, o CNPq e o CAPES. Em estudo bibliométrico de outra área odontológica, Barbosa et al. ${ }^{12}$ relataram que as instituições fomentadoras de destaque foram as FAPs e o CNPq. Isso demonstra que a falta de recursos financeiros é uma realidade para muitas pesquisas no país.

\section{CONCLUSÃO}

As áreas da endodontia e da bibliometria, assim como as áreas científicas de forma geral, estão em constante evolução. Através desse trabalho pode-se perceber que a área endodôntica é de grande interesse para o campo da pesquisa científica. Entretanto, é notável a necessidade de incentivo à pesquisa endodôntica, visto que a maioria dos trabalhos não teve financiamento e que há grande concentração de iniciativas em alguns estados e universidades. Os resultados do estudo em questão permitirão comparações com investigações futuras através dos indicadores bibliométricos.

\section{REFERÊNCIAS}

1. AAE. Glossary of Endodontic Terms. 8.ed. Chicago: American Association of Endodontists; 2012.
2. Araújo FL, Alvarenga L. A bibliometria na pesquisa científica da pós-graduação brasileira de 1987 a 2007. Enc Bibli: R Eletr Bibliotecon Ci Inf. 2011; 16(31):5170.

3. Pinheiro RC, Pizzani L, Martinez CMS, Hayashi MCPI. Produção científica sobre avaliação da visão em crianças: um estudo bibliométrico na base de dados LILACS. Rev Educ Esp. 2012;25(42):143-66.

4. Hid DS, Nascimento C, Oliveira DA. Análise das publicações internacionais relacionadas ao desenvolvimento sustentável na área de administração: uma análise bibliométrica da produção científica. RAEP. 2012; 13 (4): 653-71.

5. Scariot R, Stadler AF, Assunção CM, Pintarelli TP, Ferreira FM. A map of brazilian dental research in the last decades. Braz Oral Res. 2011; 25(3):197-204.

6. Gil-Montoya JA, Navarrete-Cortes J, Pulgar R, Santa S, Moya-Anegon F. World dental research production: an ISI database approach (1999-2003). Eur J Oral Sci. 2006; 114: 102-08.

7. Glanzel W, Leta J, Thijs B. Science in Brazil. Part I: a macro-level comparative study. Scientometrics. 2006; 67(1): 67-86.

8. Dias AA, Narvai PC, Rêgo DM. Tendências da produção científica em odontologia no Brasil. Rev Panam Salud Pública. 2008; 24(1):54-60.

9. Guedes V, Borschiver S. Bibliometria: uma ferramenta estatística para a gestão da informação e do conhecimento, em sistemas de informação, de comunicação e de avaliação científica e tecnológica. In: VI CINFORM, 6., 2005, Salvador. Anais do Encontro Nacional de Ciências da Informação. Salvador, 2005.

10. Brito Jr. M, Dias LC, Veloso DNP, Camilo CC, Martins AMEBL, Ferreira RC. Estudo bibliométrico de artigos brasileiros publicados em periódicos internacionais de Endodontia: período 2008-2010. Arq Odontol. 2011; 47(2):84-89.

11. Miller PMC. Análise das publicações científicas indexadas (JCR) no âmbito da endodontia (1998-2008). Tese [Doutorado em Odontologia] - Departamento de Estomatologia da Facultad de Medicina y Odontología, Universitat de València, Valência; 2013.

12. Barbosa VLSA, Nóbrega DRM, Cavalcanti AL. Estudo bibliométrico de pesquisas realizadas com fitoterápicos na odontologia. R bras ci Saúde. 2012; 16(2):123-30.

\section{CONFLITO DE INTERESSES}

Os autores declaram não haver conflitos de interesse.

\section{AUTOR PARA CORRESPONDÊNCIA}

\section{Diego Romário da Silva}

diegoromárioo@gmail.com

Submetido em 04/07/2017 Aceito em 10/08/2017 